\title{
Análise de Sobrevivência dos Infectados pela COVID-19 no Estado do Rio Grande do Norte
}

\author{
Gabriella da Silva Cavalcanti ${ }^{1}$ \\ ${ }^{1}$ Universidade Federal do Rio Grande do Norte. E-mail: gabriella_s.cavalcanti@hotmail.com
}

\begin{abstract}
RESUMO
O presente estudo tem por objetivo investigar os determinantes da probabilidade e do tempo de sobrevivência dos infectados pelo novo coronavírus no estado do Rio Grande do Norte. Para tanto, foram utilizados os microdados oficiais de notificações de COVID-19 disponibilizados pela Secretaria de Estado da Saúde Pública (SESAP-RN) para estimar a função de sobrevivência, a partir do método de Kaplan-Meier (1958) e do modelo de risco de Cox (1972). Uma coorte de 13.738 casos confirmados de COVID-19 no estado entre as datas de 09/03/2020 a 12/06/2020 foi analisada. Os resultados apontaram uma queda na probabilidade de sobrevivência do infectado com o passar do tempo e que a idade, a preexistência de comorbidades como hipertensão arterial sistêmica, obesidade e doenças renais crônicas, a Região de Saúde e o bairro de residência influenciam a probabilidade de sobrevivência dos infectados pela COVID-19 no Rio Grande do Norte, corroborando com as evidências internacionais.
\end{abstract}

\section{PALAVRAS-CHAVE}

COVID-19, Pandemia, Análise de sobrevivência

Survival Analysis of COVID-19 Infected Patients in the State of Rio Grande do Norte

\begin{abstract}
The present study aims to investigate the determinants of probability and survival time of those infected by the new coronavirus in the state of Rio Grande do Norte. For this purpose, the official microdata of COVID-19 notifications available by the Secretary of State for Public Health (SESAP-RN) was used to estimate the survival function, from the Kaplan-Meier method (1958) and the Cox risk model (1972). A cohort of 13,738 confirmed cases of COVID-19 in the state between 03/09/2020 and 06/12/2020 was analyzed. The results indicated a decrease in the probability of survival of the infected over time and that age, the preexisting comorbidities such as systemic arterial hypertension, obesity and chronic kidney diseases and the Region of Health and the residence district influence the probability of survival of those infected with COVID-19 in Rio Grande do Norte, corroborating international evidence.
\end{abstract}

KEYWORDS

COVID-19, Pandemic, Survival analysis

CLASSIFICAÇÃO JEL

C34, C41, I14, I18

\section{Introdução}

Desde o primeiro caso oficial de Sars-Cov-2, em dezembro de 2019, em Wuhan na China, o novo coronavírus tem causado inúmeros impactos negativos em todo o 
mundo (Wu et al., 2020; Zhou et al., 2020). Alterações da dinâmica demográfica, redução da mobilidade e das interações humanas, redução significativa da renda, produção e emprego dos países, aumento da vulnerabilidade e das desigualdades sociais e a construção de agendas políticas de securitização da saúde são alguns dos impactos previstos da pandemia de COVID-19 (Madhav et al., 2017). A inexistência de uma vacina impõe aos governantes a implementação de medidas de isolamento social aliadas à ampliação de equipamentos hospitalares, aquisição de insumos e contratação de recursos humanos visando o combate à pandemia.

Em relação aos aspectos clínicos, o amplo estudo realizado na China, com dados epidemiológicos de 72.314 casos de COVID-19, encontrou evidências de que aproximadamente $80,9 \%$ dos casos são leves, $13,8 \%$ graves e $4,7 \%$ críticos (China CDC Weekly, 2020). Os casos mais graves ocorrem com maior frequência na população idosa ou naquela diagnosticada com alguma doença preexistente (doenças cardiovasculares, diabetes, doenças respiratórias crônicas, hipertensão, etc), de acordo com os estudos empíricos (China CDC Weekly, 2020; Jin et al., 2020; Yang et al., 2020).

A literatura empírica acerca da COVID-19 ainda é muito incipiente. Em termos gerais, as evidências apontam que o sexo masculino, a idade avançada, a preexistência de comorbidades como hipertensão, doenças cardíacas, diabetes, doenças respiratórias, doença renal crônica, entre outras, o uso de medicações contínuas como estatinas, diuréticos, a hospitalização, a internação em unidade de terapia intensiva, intubação e a atenção à saúde no serviço público são fatores que aumentam o risco de morte dos infectados pelo novo coronavírus (Castelnuovo et al., 2020; Grasselli et al., 2020; Li et al., 2020; Nemati et al., 2020; Salinas-Escudero et al., 2020; Tian et al., 2020).

O Brasil, em 13 de junho de 2020, já acumulava 850.796 casos de COVID-19 e 42.791 óbitos (Ministério da Saúde, 2020b). A subnotificação - devido à baixa testagem - tão explícita e criticada no país acaba por elevar falsadamente as taxas de mortalidade e de letalidade. As taxas de letalidade e mortalidade do país atualmente são de 4,9\% e 20,9/100 mil habitantes, respectivamente, colocando o Brasil em 15음 lugar no mundo no número de mortes da doença proporcionalmente à população (Batista et al., 2020a). Além disso, a taxa de contágio estimada para o país é igual a 1,4, a terceira mais alta do mundo, atrás apenas de Porto Rico e Bangladesh e à frente dos Estados Unidos (Lichtenthäler Filho e Lichtenthäler, 2020).

A região Nordeste apresenta números acima da média nacional e possui a segunda maior taxa de mortalidade (23 mortes/ 100 mil habitantes) e letalidade $(4,54 \%)$ entre as regiões brasileiras, perdendo somente para as regiões Norte e Sudeste. O Estado do Rio Grande do Norte, por sua vez, ocupa a $3^{\circ}$ e $6^{\circ}$ colocação no Nordeste na taxa de letalidade e mortalidade por COVID-19, respectivamente, com 3,87\% e 15,2 óbitos por 100 mil habitantes, atrás apenas da média regional de 4,54\% e dos estados de Pernambuco $(8,47 \%)$ e do Ceará $(6,32 \%)$ (LAIS-UFRN, 2020). 
Em recente pesquisa, Batista et al. (2020b) sugerem que, para além das características próprias do novo coronavírus, os fatores socioeconômicos também podem ser determinantes para explicar a evolução do quadro clínico de um paciente contaminado pela COVID-19 no Brasil. Foi observada uma taxa maior de letalidade em indivíduos idosos, negros e pardos, sem escolaridade, residentes em municípios com baixo Índice de Desenvolvimento Humano (IDHM) e internados em Unidades de Tratamento Intensivo (UTI's). A pesquisa ainda aponta que a letalidade entre autodeclarados negros e pardos é superior à dos brancos, mesmo considerando a faixa etária e nível de escolaridade, sugerindo que a taxa de letalidade é afetada pelas desigualdades de acesso ao tratamento.

No mesmo sentido, as evidências encontradas por Santos et al. (2020) mostram que a COVID-19 é mais letal na periferia do país devido ao adensamento populacional, ausência de saneamento básico e escassez na oferta de serviços de saúde. A pandemia, que começou a se disseminar no Brasil primeiro pelas rotas aéreas entre as capitais e principais centros urbanos, depois por vias rodoviárias que interligam os municípios, chega posteriormente na periferia das grandes cidades que depende exclusivamente da rede pública de saúde (Delatorre et al., 2020; Fio Cruz, 2020; Noronha et al., 2020; Santos et al., 2020).

Ao analisarem o intervalo de tempo entre o início de sintomas gripais e a realização do teste para COVID-19 nas capitais brasileiras e no Distrito Federal, Lima et al. (2021) encontraram que esse tempo médio foi de 10,2 dias, predominando a testagem nos indivíduos do sexo feminino, na faixa etária de 20-39 anos e da região Sudeste. Ademais, o estudo concluiu pela inadequação no tempo de realização dos testes para COVID-19 nas regiões brasileiras.

Com o objetivo de investigar se o delay no diagnóstico representa um fator determinante da mortalidade de pacientes com COVID-19 no Rio de Janeiro, Cobre et al. (2020) estimaram funções Kaplan-Meier, modelos de regressão Cox e regressões logísticas multivariadas. Os autores encontraram que o tempo mediano do início dos sintomas ao diagnóstico foi de 8 dias e que as taxas de mortalidade foram maiores em homens, idosos e em pacientes que residem em bairros com baixo índice de desenvolvimento social. Os resultados apontaram que atrasos no diagnóstico superiores a 8 dias configuram fatores de risco para o óbito de pacientes infectados pelo novo coronavírus.

A associação entre a desigualdade econômica e o risco de infecção e morte por COVID-19 foi o tema do estudo realizado por Demenech et al. (2020). Os autores aplicaram testes de correlação e de dependência espacial e encontraram evidências de correlação positiva entre o coeficiente de Gini e as taxas de incidência e mortalidade pelo novo coronavírus nas unidades federativas, que manteve-se mesmo ao controlar pelas variáveis demográficas e espaciais. Concluiu-se, então, que a desigualdade econômica exerce um papel determinante no impacto da COVID-19 nos estados brasileiros. 
Dentro desse contexto, esse estudo se justifica pela contribuição que pretende dar a literatura ao se dedicar a entender o padrão de letalidade da doença numa área menos privilegiada do país, de modo a fornecer informações estratégicas aos elaboradores de políticas públicas para reduzir o número de mortes. O presente estudo pretende investigar se as características individuais e regionais podem ajudar a explicar a sobrevivência dos infectados pelo novo coronavírus no estado do Rio Grande do Norte (RN). Como exposto na próxima seção, esse estado é caracterizado por possuir indicadores socioeconômicos mais vulneráveis que a média nacional. Adicionalmente, conforme os Boletins Epidemiológicos divulgados pela Secretaria de Estado da Saúde Pública do Rio Grande do Norte (SESAP-RN), a taxa de ocupação dos leitos destinados para o tratamento dos pacientes acometidos pelo novo coronavírus está próximo da capacidade máxima, o que intensifica o risco do colapso dos sistemas de saúde.

O objetivo desse estudo é, então, estimar a probabilidade e o tempo de sobrevivência dos infectados pela COVID-19 no estado do RN condicionados às características individuais, condição de saúde e Região de Saúde do indivíduo. Foi feito também um recorte amostral para a cidade de Natal visando condicionar a probabilidade de sobrevivência do infectado ao seu bairro de residência. Uma coorte de 13.738 casos confirmados de COVID-19 no estado entre as datas de 09/03/2020 a 12/06/2020 foi analisada utilizando da técnica de análise de sobrevivência por meio da estimação da função de sobrevivência através do método Kaplan e Meier (1958) e do modelo de risco de Cox (1972), a exemplo do que foi realizado por Nemati et al. (2020) e Tian et al. (2020) para o caso da China.

O presente estudo se encontra estruturado em mais quatro seções além desta introdução. A seção 2 discorre acerca de um breve histórico das principais pandemias respiratórias. A seção 3 oferece a caracterização do estado do Rio Grande do Norte. A seção 4, por sua vez, descreve a técnica de Análise de Sobrevivência e os dados utilizados. As seções 5 apresenta e discute os resultados e, por fim, a seção 6 apresenta as principais conclusões do estudo.

\section{Breve histórico das principais pandemias respiratórias}

As pandemias são surtos em larga escala de doenças infecciosas que podem aumentar muito a mortalidade em uma ampla escala geográfica e causar perturbações econômicas, sociais e políticas significativas (Madhav et al., 2017; Senhoras, 2020) As evidências empíricas apontam que a probabilidade de ocorrência de pandemias aumentou ao longo do século passado por conta do aumento da circulação de pessoas e globalização, urbanização, mudanças no uso da terra e maior exploração do meio ambiente (Jones et al., 2008; Morse, 1995).

As pandemias mais notáveis e com amplas repercussões espaço-temporais datam do século VI com a conhecida "Praga do Justiniano", passando pela "Peste Negra"no século XIV, até se chegar à "Gripe Espanhola"e "Gripe Asiática"no século XX. No início 
do século XXI surgiram diversas pandemias respiratórias como a Síndrome Respiratória Aguda Grave (SARS), a "Gripe Suína"e a Síndrome Respiratória do Oriente Médio (MERS), culminando com a recente pandemia do COVID-19.

Diversos são os impactos econômicos, sociais e políticos das pandemias. Os impactos diretos se dão na saúde dadas as perdas de vidas em decorrência das pandemias. Estima-se que a Peste Negra resultou na morte de 30\% a 50\% da população européia (DeWitte, 2014) e que a Gripe Espanhola em 1918 causou de 20 a 100 milhões de mortes, mais mortes do que a Primeira Guerra Mundial que ceifou a vida de $17 \mathrm{mi}$ lhões de pessoas (Johnson, 2002). Os impactos indiretos na saúde advém do aumento da morbidade e mortalidade devido à interrupção dos tratamentos eletivos. Durante o surto de Ebola na África, estima-se que a interrupção do tratamento de rotina de doenças como a malária, HIV/AIDS e tuberculose tenha causado 10,6 mil mortes no continente (Parpia et al., 2016).

Acerca dos impactos econômicos, as pandemias ocasionam, inicialmente, choques fiscais de curto prazo gerados pelos gastos de contenção da pandemia, como a construção de hospitais, compra de insumos e equipamentos hospitalares, contratação de profissionais de saúde, etc e também pela redução na arrecadação tributária. Há ainda os efeitos indiretos na atividade e no crescimento econômico causados pela redução da força de trabalho devido ao adoecimento e morte, além de mudanças comportamentais dos agentes provocadas pelo medo de contaminação e medidas de isolamento como lockdowns.

Durante a Gripe Espanhola, estima-se que ocorreu uma redução do PIB de 11\% nos Estados Unidos, 15\% no Canadá e 17\% no Reino Unido (Mckibbin, 2006). As pandemias de H1N1 e de MERS, causaram uma redução em U\$\$ 1 bilhão e U\$\$ 2 bilhões no PIB da República da Coréia, respectivamente, e esta última ainda provocou uma despesa de U\$\$ 14 bilhões em estímulos governamentais (Madhav et al., 2017). Segundo estimativas do Fundo Monetário Internacional (FMI), espera-se que a pandemia de COVID-19 cause uma perda global da ordem de U\$\$ 12 trilhões de dólares (FMI, 2020).

\section{Caracterização do Rio Grande do Norte}

O estado do Rio Grande do Norte (RN), situado na Região Nordeste do Brasil, possui características populacionais, demográficas e socioeconômicas que, combinadas, podem contribuir para o colapso do sistema de saúde e, consequentemente, para o aumento da taxa de mortalidade da COVID-19. Os dados do último Censo Demográfico realizado no Brasil revelam que, em 2010, esse estado apresentou indicadores sociais mais preocupantes que a média nacional, como a baixa renda per capita $(\mathrm{R} \$ 545,42$ contra $\mathrm{R} \$ 793,87$ do Brasil) e alta taxa de pobreza (23,79\% contra $15,20 \%)$ (IBGE, 2010). O Índice de Desenvolvimento Humano (IDH - 2010) do RN é igual a 0,684, o $16^{\circ}$ do país, sendo classificado como médio desenvolvimento. 
Os estudos clínicos sugerem que, além dos idosos, as pessoas com alguma comorbidade são mais susceptíveis a desenvolverem o estado grave de COVID-19, aumentando a probabilidade de evoluir para óbito (China CDC Weekly, 2020; Jin et al., 2020; Yang et al., 2020). Em 2010, aproximadamente 10,8\% da população potiguar tinha idade igual ou maior que 60 anos, sendo o sétimo estado brasileiro com maior proporção idoso (IBGE, 2010).

Em relação às comorbidades, a Pesquisa Nacional de Saúde (PNS) realizada pelo IBGE em 2019 indica que no estado há um elevado percentual de pessoas com idade acima de 18 anos diagnosticadas com pelo menos uma doença crônica, sendo que $21,9 \%, 8,8 \%, 4,8 \%, 4,5 \%$ delas com hipertensão arterial, diabetes, asma e doenças cardíacas, respectivamente (IBGE, 2021).

Além disso, o estado apresenta forte heterogeneidade espacial. De acordo com os dados do Censo 2010, a maior parte da população idosa reside no interior do estado, onde a renda per capita é inferior à média estadual, $\mathrm{R} \$ 298,17$ e $\mathrm{R} \$ 545,42$, respectivamente, tornando-a ainda mais vulnerável (IBGE, 2010). Em contrapartida, na região metropolitana e capital, há uma maior concentração de domicílios adensados (8,8\%), localizados principalmente nas periferias, onde há menor acesso ao abastecimento de água encanada e esgotamento sanitário, aumentando o risco de contágio nessas localidades.

No que se refere à infraestrutura da saúde para o enfrentamento da doença, os dados do Cadastro Nacional de Estabelecimento de Saúde- CNES (2020) mostram que, ao longo do primeiro semestre de 2020, foram implementados leitos específicos para o tratamento dos pacientes infectados pelo novo coronavírus no estado Ministério da Saúde (2020a). Em junho de 2020, havia 699 leitos de UTI II Adulto (aumento de 129\% quando comparado ao mês de fevereiro de 2020). Do total desses leitos, 394 foram destinados para os pacientes acometidos pela COVID-19. Contudo, a Figura 1 ilustra que os leitos de UTI são desigualmente distribuídos entre as oito Regiões de Saúde (RS) do estado ${ }^{1}$.

Aproximadamente 58\% dos leitos de UTIs estavam localizados na $7^{\text {a }}$ RS (Metropolitana), região que abriga a capital - epicentro da doença no estado - e que concentra 55,6\% do total de casos de COVID-19 do RN. Natal, capital do RN, concentra 38\% dos casos de COVID-19 registrados e também 46\% dos leitos de UTI do Estado. Além disso, verifica-se uma distribuição espacial desigual de equipamentos hospitalares, leitos e respiradores na própria capital do Estado (IPEA, 2020). De maneira oposta, até o momento, não foi destinada nenhuma UTI para $8^{\text {a }}$ (Vale do Açu) RS, ainda que essa região tenha contabilizado $4,0 \%$ dos casos até 13 de junho de 2020.

\footnotetext{
${ }^{1}$ As Regiões de Saúde (RS) são "espaços geográficos contínuos constituídos por agrupamentos de Municípios limítrofes [...], com a finalidade de integrar a organização, o planejamento e a execução de ações e serviços de saúde"(Ministério da Saúde, Resolução n. 1, de 29 de setembro de 2011)
} 
Figura 1. Regiões de Saúde do RN e distribuição dos leitos de UTI II ADULTO e dos casos de COVID-19.

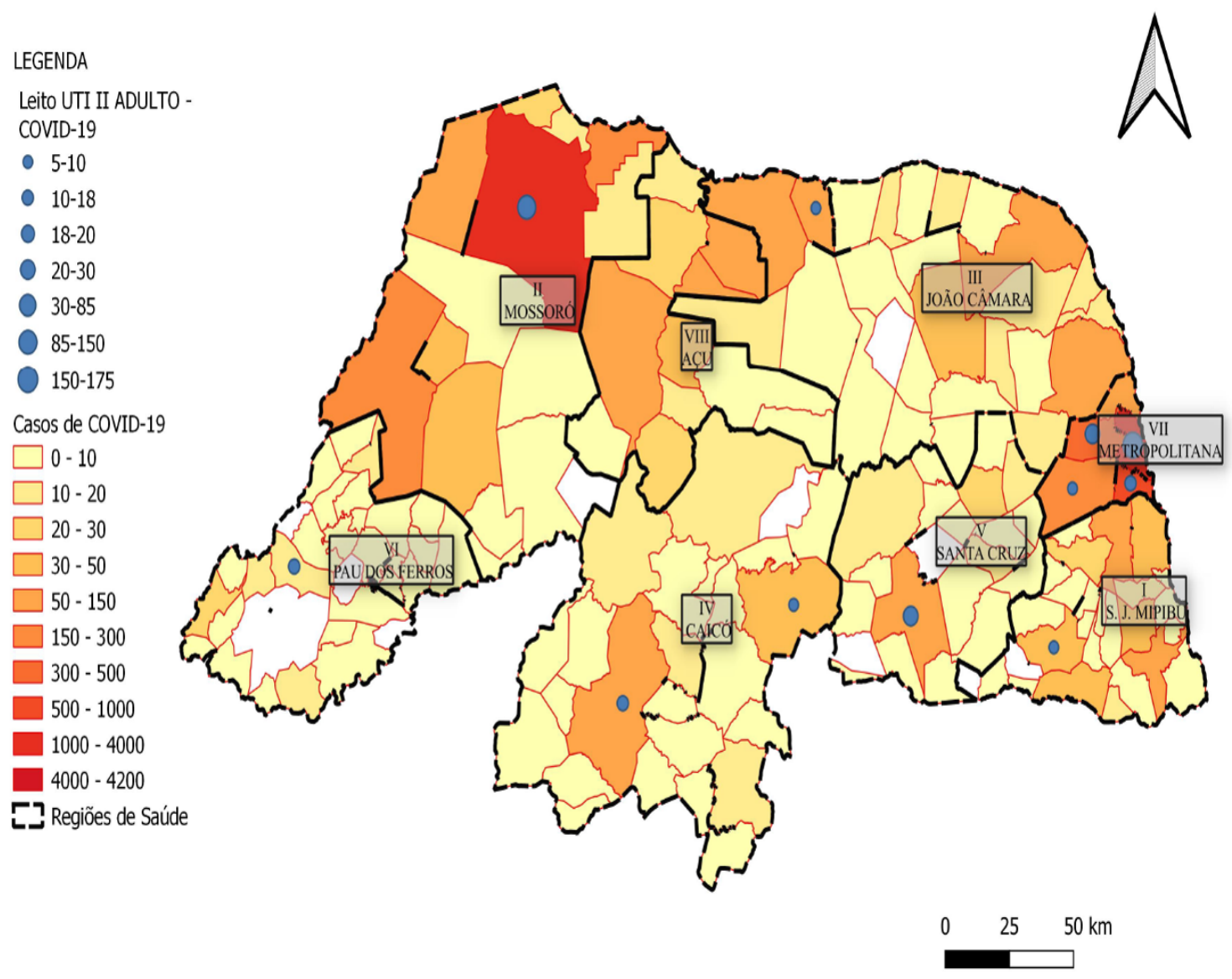

Fonte: Elaboração própria com base nos dados da SESAP-RN.

Nota: Dados extraídos em 13 de junho de 2020, sujeitos a atualizações.

A Tabela 1 a seguir dá um panorama mais detalhado do sistema de saúde por Região de Saúde do estado. Como pode-se verificar da Tabela 1, a estrutura física e humana do sistema de saúde do estado está fortemente concentrada na RS 2 Mossoró e na RS 7 - Metropolitana, que juntas detêm, majoritariamente, os leitos de UTI, enfermarias, respiradores e equipes de saúde do RN.

A despeito da inauguração de leitos específicos para o tratamento dos pacientes com COVID-19, eles parecem não ser suficiente para atender a demanda da população infectada, isso porque o sistema de saúde do estado está próximo do colapso, sendo que a taxa de ocupação já atingiu a capacidade máxima em Natal e Mossoró, segundo a SESAP-RN. Até o dia 13 de Junho de 2020, foram confirmados 13.789 casos e 533 óbitos pela doença no estado (Rio Grande do Norte, 2020). Dos 167 municípios potiguares, 156 deles já apresentaram pelo menos um caso e 74 confirmaram a ocorrência de óbitos, o que sugere o espraiamento da doença por todo território potiguar. 
Tabela 1. Estrutura física e de recursos humanos do sistema de saúde segundo as Regiões de Saúde do Rio Grande do Norte

\begin{tabular}{|c|c|c|c|c|c|c|c|}
\hline Regiões de Saúde & $\begin{array}{c}\text { Leitos de } \\
\text { UTI } \\
\text { Adulto II } \\
- \\
\text { Covid-19 }\end{array}$ & $\begin{array}{l}\text { Enfer- } \\
\text { marias }\end{array}$ & $\begin{array}{l}\text { Respira- } \\
\text { dores }\end{array}$ & Médicos & $\begin{array}{l}\text { Enfermei- } \\
\text { ros }\end{array}$ & $\begin{array}{l}\text { Fisiotera- } \\
\text { peutas }\end{array}$ & $\begin{array}{l}\text { Técnicos } \\
\text { auxilia- } \\
\text { res de } \\
\text { enferma- } \\
\text { gem }\end{array}$ \\
\hline I RS - São José do Mipibu & 5 & 433 & 15 & 293 & 336 & 101 & 489 \\
\hline II RS - Mossoró & 85 & 1115 & 191 & 644 & 554 & 186 & 1223 \\
\hline III RS - João Câmara & 20 & 372 & 19 & 221 & 342 & 68 & 641 \\
\hline IV RS - Caicó & 35 & 714 & 43 & 330 & 328 & 92 & 785 \\
\hline V RS - Santa Cruz & 22 & 378 & 26 & 174 & 221 & 78 & 404 \\
\hline VI RS - Pau dos Ferros & 11 & 880 & 28 & 213 & 289 & 97 & 699 \\
\hline VII RS Metropolitana & 246 & 3582 & 700 & 3243 & 1913 & 425 & 5407 \\
\hline VIII RS - Açu & 10 & 176 & 5 & 132 & 147 & 39 & 295 \\
\hline Total & 434 & 7650 & 1027 & 5250 & 4130 & 1086 & 9583 \\
\hline
\end{tabular}

Fonte: Elaboração própria a partir dos dados do DataSUS (2020).

Nota: Dados obtidos em 13 de junho de 2020, sujeitos a atualizações.

\section{Metodologia}

\subsection{Análise de sobrevivência}

Os modelos de duração têm suas origens na técnica de Análise de Sobrevivência. A Análise de Sobrevivência ou Análise de Sobrevida consiste em um conjunto de procedimentos estatísticos em que a variável de interesse é o tempo que decorre até a ocorrência de um determinado evento ou falha, mais denominada de tempo de sobrevivência (Cavalcanti, 2020; Kleinbaum e Klein, 2012; Wooldridge, 2002). No caso do presente estudo, a variável de interesse é o tempo em dias entre a data de início de sintomas e a data de evolução, isto é, o tempo decorrido até o paciente infectado pela COVID-19 evoluir a óbito.

A análise de sobrevivência é comumente utilizada na literatura empírica quando ocorre o problema de dados censurados, isto é, quando não se conhece exatamente o tempo de sobrevivência de algum (ns) indivíduo (s) da amostra (Cavalcanti, 2020; Greene, 2008; Kleinbaum e Klein, 2012). Quando o tempo verdadeiro de sobrevivência é maior do que o tempo de sobrevivência observado têm-se dados censurados à direita. No caso dos dados de infectados pela COVID-19, ocorre a censura à direita devido à limitação de atualização dos dados até a data fim do estudo, 13 de junho de 2020, não permitindo conhecer exatamente o tempo de sobrevivência daqueles pacientes que ainda estão internados em enfermarias, UTI's ou estão em tratamento domiciliar da doença até a data de 12 de junho de 2020, data da última notificação de infectado pela COVID-19 que consta na base de dados disponibilizada pela SESAP-RN 
em 13/06/2020. A análise compreendeu uma coorte de 13.738 casos confirmados de COVID-19 no estado entre as datas de 09/03/2020 a 12/06/2020.

Nos modelos de análise de dados de sobrevivência, o arcabouço matemático é dado pela função de sobrevivência, $S(t)$, e pela função de risco $h(t)$. Denotando $T$ como uma variável aleatória que representa o tempo de sobrevivência de um dado indivíduo e $t$ como um valor específico do tempo $T$, consoante Kleinbaum e Klein (2012), a função de sobrevivência $S(t)$ representa a probabilidade de que $T$ exceda $t$, isto é $S_{(t)}=P(T \geq t)$. Existindo observações censuradas, torna-se necessário um método alternativo para calcular a probabilidade de sobrevivência, denominado estimador de Kaplan-Meier (Kleinbaum e Klein, 2012).

Segundo Kaplan e Meier (1958), à priori, o estimador exige a ordenação decrescente dos tempos de sobrevivência $t$ até a ocorrência do evento ou falha $f$. A fórmula KaplanMeier de probabilidade de sobrevivência pode ser demonstrada como na Equação 1 que segue:

$$
\hat{S}\left(t_{(f)}\right)=\hat{S}\left(t_{(f-1)}\right) \times \hat{\operatorname{Pr}}\left(T>t_{(f)} \mid T \geq t_{(f)}\right)
$$

esta fórmula mensura a probabilidade de sobreviver após o tempo anterior de falha $t_{(f-1)}$, multiplicada pelo probabilidade condicional de sobreviver passado o tempo $\left.t_{(} f\right)$, dada a sobrevivência até pelo menos o tempo $\left.t_{(} f\right)$. Ao substituir a probabilidade de sobrevivência $\hat{S}\left(t_{(f-1)}\right)$ pelo produto-limite, em outras palavras, o produto de todas as probabilidades condicionais para os tempos de falha $t_{(f-1)}$ e anteriores, obtêm-se a Equação 2 a seguir:

$$
\hat{S}\left(t_{(f-1)}\right)=\prod_{i=1}^{f-1} \hat{\operatorname{Pr}}\left(T>t_{(i)} \mid T \geq t_{(i)}\right)
$$

A principal vantagem do estimador de Kaplan e Meier (1958), de acordo com Greene (2008), consiste no fato de tratar-se de uma abordagem não-paramétrica que dispensa uma especificação para a função de risco na maioria das vezes desconhecida pelo pesquisador, além de assumir tempos de sobrevivência independentes e identicamente distribuídos, de modo a eliminar a possibilidade de dependência de duração.

A Função de Risco $h(t)$ denota a probabilidade do evento ocorrer por unidade de tempo em um pequeno intervalo de tempo, $\Delta t$, dada a sobrevivência do indivíduo até o tempo $t$, significando assim, o oposto da função de sobrevivência. A Equação 3 na sequência modela a função de risco:

$$
h(t)=\lim _{\Delta t \rightarrow 0} \frac{P(t \leq T \leq t+\Delta t \mid T \geq t)}{\Delta t} t
$$

Visando estimar a correlação entre o tempo de sobrevivência do infectado pela 
COVID-19, caracteristicamente censurado, e as covariadas, torna-se necessário a estimação de um modelo de regressão específico chamado Regressão Cox. O modelo matemático comumente utilizado na literatura empírica constitui a abordagem de risco proporcional de Cox (1972) expresso em termos do risco no tempo $t$ para um indivíduo com uma determinada especificação para o vetor de covariadas $X$, como na Equação 4 abaixo:

$$
h(t, X)=h_{0}(t) e^{\sum_{i=1}^{p} \beta_{i} X_{i}}
$$

na qual $h_{0}(t)$ é a função de risco baseline, que assume que todas as variáveis $X s$ são iguais a zero, e o termo $e^{\sum_{i=1}^{p} \beta_{i} X_{i}}$ é o exponencial do somatório de $\beta_{i} X_{i}$ das $p$ variáveis independentes $X$ do modelo.

Os coeficientes do modelo de risco proporcional de Cox foram estimados através do Método de Máxima Verossimilhança (MV) baseado na maximização de uma função de probabilidade parcial, denominada assim porque considera apenas os indivíduos que sofrem o evento durante o período analisado, ou seja, os não-censurados.

Sendo assim, a estimação do modelo de duração da sobrevivência utilizado no presente estudo é obtido conforme a Equação 5 a seguir:

$$
\text { durao_sobrevivncia }=X \beta
$$

em que $X$ é o vetor de variáveis explicativas contínuas de idade e tempo de diagnóstico e variáveis dummy de sexo, cor, tipos de comorbidades e Região de Saúde de residência do infectado.

Visando analisar a correlação entre tipos específicos de comorbidades e a probabilidade de óbito, foram criadas e inseridas dummies no modelo a partir da atribuição do valor 1 quando da preexistência de determinada comorbidade no indivíduo infectado e 0 , caso contrário. Foram estimados vários modelos, chegando aos mais robustos e apresentados na seção de resultados. O nivel de significância considerado para avaliar as covariadas foi de $5 \%$.

Procedeu-se ainda da realização de testes de diagnóstico da influência de outliers a partir do exame dos resíduos do modelo Cox. Para fins de estimação, foi utilizado o software estatístico R versão 3.6.1 (R Core Team, 2019).

\subsection{Base de dados e estatísticas descritivas}

A fonte dos dados utilizada no presente estudo é proveniente dos microdados de notificações de COVID-19 divulgados no Boletim Epidemológico № 87, em 13 de Junho 
de $2020^{2}$, pela SESAP-RN (Rio Grande do Norte, 2020).

Para atingir os objetivos propostos, foram considerados apenas os casos confirmados de COVID-19 no Estado do Rio Grande do Norte e na cidade de Natal/RN. Dois períodos diferentes de observação do infectado foram observados: um referente à data de início dos sintomas declarada pelo indivíduo e outro referente à data de evolução do estado de saúde do infectado, que pode se referir à data de cura, de óbito ou declarando que o paciente ainda se encontra internado em enfermarias ou UTI's ou ainda em tratamento domiciliar da doença.

Para construção da variável de tempo da sobrevivência, foram contabilizados os dias entre a data de sintomas e a de óbito. Provavelmente, em decorrências de erros de notificação, em alguns casos foram atribuídos tempo de sobrevivência negativo. Esses casos foram eliminados da amostra por considerar inconsistente o fato do evento óbito acontecer antes do início dos sintomas.

Posteriormente, para identificar a ocorrência do evento morte, foi criada uma variável dummy a qual foi atribuído valor 1 em caso de óbito do infectado e 0 para as censuras, isto é, em caso de não ocorrência do evento óbito até o fim do estudo para os indivíduos recuperados, internados, em tratamento domiciliar da COVID-19 e para aqueles sem informação.

Adicionalmente, foram incorporadas no estudo variáveis que identificam o sexo, a cor, a idade, doenças preexistentes, tipos de comorbidades, tempo de diagnóstico, Regiões de Saúde e o bairro de residência do infectado, conforme as estatísticas descritivas apresentadas na Tabela 2. A amostra final foi de 13.738 casos confirmados de COVID-19 no Estado do Rio Grande do Norte.

Em relação ao tempo médio de diagnóstico e de sobrevivência, esses são de 7,4 e 12, 1 dias, respectivamente, para os pacientes que vieram a óbito. O menor tempo de diagnóstico encontrado para o grupo de pacientes que faleceram pode ser resultado da política que, diante da escassez de testes para a COVID-19, priorizou a testagem nas pessoas que pertenciam ao grupo de risco e nos pacientes sintomáticos.

Os infectados pela COVID-19 no RN têm, em média, 43,6 anos, ao passo que a média de idade dos pacientes que vieram a óbito é de 66,6 anos. Apesar da amostra dos infectados apresentar-se equilibrada em relação ao sexo dos indivíduos, a amostra de óbitos é composta majoritariamente por $58 \%$ de indivíduos do sexo masculino. Quanto à cor/raça/etnia, vê-se que 50,2\% dos infectados se autodeclararam pardos e negros, assim como a maioria dos que faleceram $(59,5 \%)$.

\footnotetext{
${ }^{2}$ Trata-se do último dado oficial do RN sobre o COVID-19 divulgado até o momento em que a atual versão desse artigo está sendo elaborada. É importante ressaltar que os dados utilizados nesse estudo estão sujeitos a atualizações, isso porque o estado do RN ainda não vivenciou o pico de contágio da doença. Soma-se a isso, a possibilidade de conter casos de subnotificação e/ou erros de notificação.
} 
Tabela 2. Estatísticas descritivas

\begin{tabular}{|c|c|c|}
\hline \multicolumn{3}{|c|}{ Rio Grande do Norte } \\
\hline Variáveis & Amostra Completa & Óbitos \\
\hline Número de casos & 13.738 & 528 \\
\hline $\begin{array}{l}\text { Tempo de sobrevivência } \\
\text { (dias) }\end{array}$ & - & 12,1 \\
\hline $\begin{array}{l}\text { Tempo de diagnóstico } \\
\text { (dias) }\end{array}$ & 9,7 & 7,4 \\
\hline Idade (anos) & 43,6 & 66,6 \\
\hline Homem & $49,5 \%$ & $58,0 \%$ \\
\hline \multicolumn{3}{|c|}{ Cor/raça/etnia } \\
\hline Branca & $33,2 \%$ & $26,4 \%$ \\
\hline Amarela & $16,5 \%$ & $14,2 \%$ \\
\hline Indígena & $0,01 \%$ & $0,00 \%$ \\
\hline Parda & $46,8 \%$ & $56,4 \%$ \\
\hline Preta & $3,4 \%$ & $3,1 \%$ \\
\hline \multicolumn{3}{|c|}{ Doenças preexistentes } \\
\hline Com comorbidade & $21,3 \%$ & $68,4 \%$ \\
\hline Quantidade de comorbidades & 0,3 & 1,2 \\
\hline Cardíacas crônicas & $12,0 \%$ & $41,9 \%$ \\
\hline Diabetes & $8,7 \%$ & $39,6 \%$ \\
\hline Respiratórias crônicas & $3,2 \%$ & $7,2 \%$ \\
\hline Renais crônicas & $1,1 \%$ & $7,6 \%$ \\
\hline Imunossupressão & $0,9 \%$ & $2,7 \%$ \\
\hline Hipertensão Arterial & $0,7 \%$ & $16,5 \%$ \\
\hline \multicolumn{3}{|l|}{ Sistêmica (HAS) } \\
\hline Obesidade & $0,4 \%$ & $6,8 \%$ \\
\hline Gestante de alto risco & $0,4 \%$ & $0,0 \%$ \\
\hline Cromossômicas & $0,2 \%$ & $0,4 \%$ \\
\hline \multicolumn{3}{|c|}{ Região de Saúde } \\
\hline Região de Saúde 1 & $5,9 \%$ & $5,9 \%$ \\
\hline Região de Saúde 2 & $20,3 \%$ & $25,2 \%$ \\
\hline Região de Saúde 3 & $6,7 \%$ & $7,6 \%$ \\
\hline Região de Saúde 4 & $2,9 \%$ & $2,5 \%$ \\
\hline Região de Saúde 5 & $2,9 \%$ & $3,0 \%$ \\
\hline Região de Saúde 6 & $1,6 \%$ & $2,3 \%$ \\
\hline Região de Saúde 7 & $55,6 \%$ & $47,3 \%$ \\
\hline Região de Saúde 8 & $4,0 \%$ & $6,3 \%$ \\
\hline \multicolumn{3}{|c|}{ Natal/RN } \\
\hline \multicolumn{3}{|c|}{ Bairros por Zona } \\
\hline Zona Norte(1) & $28,8 \%$ & $39,0 \%$ \\
\hline Zoa Sul(2) & $27,5 \%$ & $16,3 \%$ \\
\hline Zona Leste(3) & $23,1 \%$ & $19,2 \%$ \\
\hline Zona Oeste(4) & $20,7 \%$ & $25,5 \%$ \\
\hline
\end{tabular}

Fonte: Elaboração própria a partir dos microdados da SESAP-RN.

Nota: Dados obtidos em 13 de junho de 2020, sujeitos a atualizações.

(1) Compõem a zona norte os seguintes bairros: Igapó, Salinas, Potengi,Nossa Senhora da Apresentação, Lagoa Azul, Pajuçara e Redinha.

(2) Compõem a zona sul os seguintes bairros: Lagoa Nova, Neópolis, Candelária, Ponta Negra,

Capim Macio, Pitimbu e Nova Descoberta.

(3) Compõem a zona leste os seguintes bairros: Santos Reis, Areia Preta, Rocas, Mãe Luíza, Ribeira, Alecrim, Praia do Meio, Barro Vermelho, Cidade Alta, Tirol, Lagoa Seca e Petrópolis.

(4) Compõem a zona oeste os seguintes bairros: Quintas, Nordeste, Felipe Camarão, Dix-Sept Rosado, Cidade da Esperança, Bom Pastor, Planalto, Nossa Senhora de Nazaré, Cidade Nova e Guarapes. 
Quanto à preexistência de doenças, 21,3\% dos pacientes foram diagnosticados com pelo menos um tipo comorbidade. Observa-se a incidência maior de doenças preexistentes entre os óbitos $(68,4 \%)$, em especial as doenças cardíacas crônicas $(41,9 \%)$, diabetes $(39,6 \%)$, hipertensão arterial sistêmica $(16,5 \%)$ e doenças renais crônicas $(7,6 \%)$. Em média, os infectados que vieram a óbito foram diagnosticados anteriormente com 1,2 doenças e o maior número de doenças por pacientes foi igual a 4 .

No que diz respeito à Região de Saúde a qual o infectado pertence, a maioria dos casos confirmados são oriundos da RS 7- Metropolitana - $(55,6 \%)$, seguido pela RS 2- Mossoró - (20,3\%). Por outro lado, a RS 6- Pau dos Ferros apresentou a menor incidência da doença $(1,6 \%)$.

Em relação aos casos confirmados na Cidade de Natal, a maioria dos infectados residem em bairros das zonas norte e sul, ambos com cerca de $28 \%$ dos casos confirmados, no entanto, é da zona norte o maior percentual de infectados pela COVID-19 que vieram a óbito (39\%).

\section{Resultados}

Essa seção tem como objetivo discutir a potencial relação entre a probabilidade de sobrevivência de um paciente acometido pela COVID-19 e as características individuais e regionais, a partir da estimação de funções de sobrevivência condicionadas ao sexo, cor, idade, condição de saúde e região de residência do infectado.

É consenso entre os estudiosos que os idosos são os mais vulneráveis à doença (Batista et al., 2020a; China CDC Weekly, 2020; Jin et al., 2020; Yang et al., 2020). O diagnóstico e tratamento da doença nessa faixa etária deve ser rápido e eficiente, de modo a evitar o agravamento da doença e, consequentemente, a morte do infectado. Essa evidência também se repete no Rio Grande do Norte. Nesse estado, os indivíduos infectados com idade igual ou maior a 60 anos apresentam, em todo o período analisado, a menor probabilidade e tempo de sobrevivência na comparação com as demais faixas etárias (Figura 2). Por outro lado, os pacientes com idade de 0 e 20 anos, crianças e adolescentes, possuem probabilidade maior de sobrevivência. Estes resultados são confirmados pelo teste Log-rank que aponta para a rejeição da hipótese nula de igualdade de probabilidade de sobrevivência ao nível de $1 \%$ de significância (p-valor de 0,0001), portanto, há diferença estatisticamente significativa nas chances de sobrevivência entre as faixas etárias.

A mediana do tempo de sobrevivência, que ocorre quando a probabilidade de sobrevivência é de 50\%, reforça a desigualdade de probabilidade entre as faixas etárias. A mediana do tempo de sobrevivência dos paciente idosos é de apenas 11 dias, inferior àquela observada para os mais novos (19 dias).

A inclinação negativa da função de probabilidade de sobrevivência pode ser explicada dado que à medida que os dias passam, os sintomas tendem a se agravar levando 
à morte do infectado.

Figura 2. Probabilidade de sobrevivência do infectado de acordo com a faixa etária.

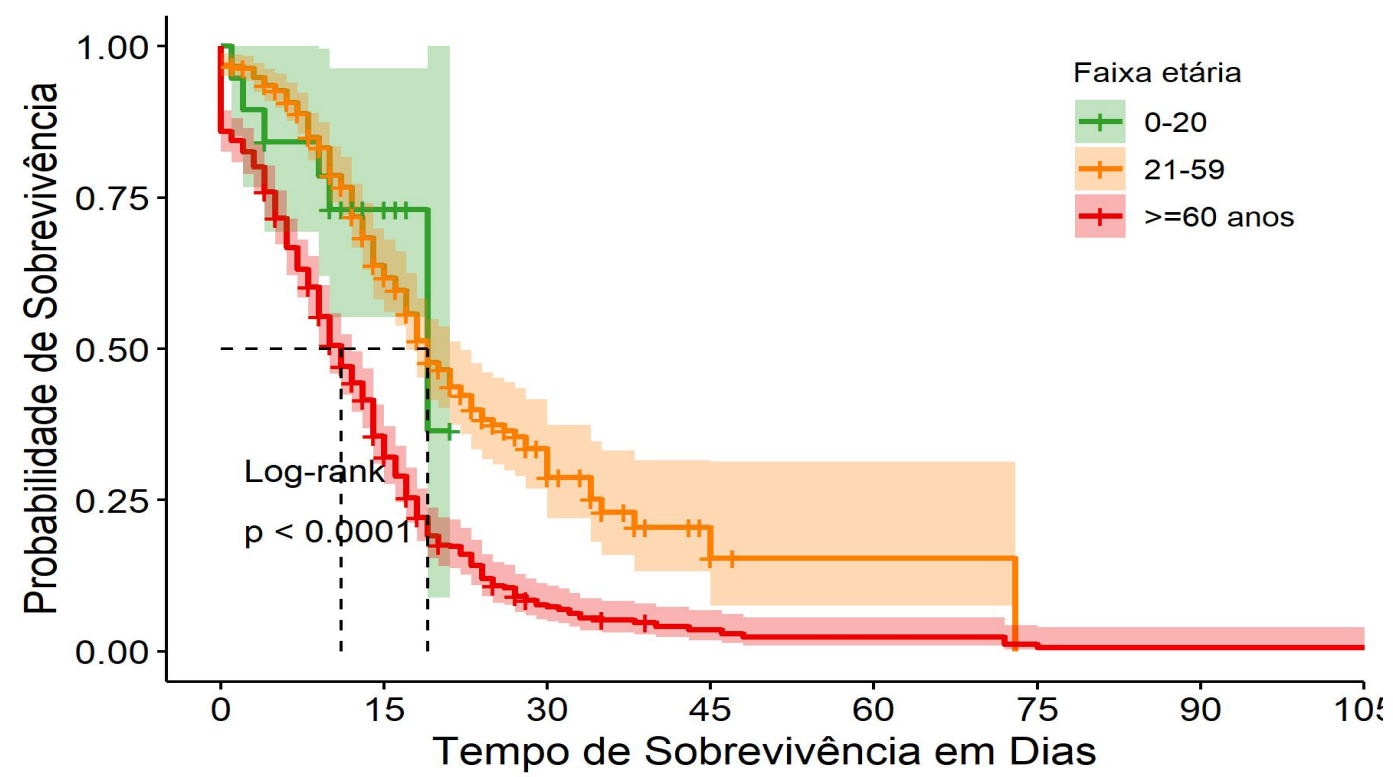

Fonte: Elaboração própria a partir dos microdados da SESAP-RN.

Nota: Dados obtidos em 13 de junho de 2020, sujeitos a atualizações.

De acordo com pesquisadores e especialistas na área da saúde, a preexistência de comorbidades no infectado pela COVID-19 consiste em um fator de risco à sobrevivência do mesmo, visto que tendem a promover o agravamento da doença no organismo desse paciente (Batista et al., 2020a; China CDC Weekly, 2020; Yang et al., 2020; Tian et al., 2020). Os casos mais críticos da doença geralmente ocorrem nos pacientes diagnosticados com hipertensão, diabetes ou cardiopatias, entre outras (China CDC Weekly, 2020; Yang et al., 2020; Tian et al., 2020). Com o auxílio da Figura 3, pode-se analisar a correlação entre a preexistência de comorbidades e a probabilidade de sobrevivência do indivíduo no Rio Grande do Norte.

Em todo o tempo de análise, é evidente o distanciamento entre a curva de sobrevivência dos pacientes diagnosticados com alguma doença crônica e a curva dos demais, indicando que a probabilidade de sobrevivência dos pacientes sem comorbidades é superior à das pessoas com comorbidades. A mediana do tempo de sobrevivência dos indivíduos com comorbidade é de apenas 11 dias, ao passo que a dos indivíduos sem comorbidades é de 32 dias.

A diferença de probabilidade de sobrevivência entre os dois grupos é corroborada pelo resultado do teste Log-rank que sugere a rejeição da hipótese nula, ao nível de $1 \%$ de significância estatística, de que as probabilidades são iguais. Dessa forma, o resultado está alinhado com as evidências científicas que colocam as pessoas diagnosticadas com doenças associadas no grupo de risco, já que essas últimas apresentam 
uma menor probabilidade de sobrevivência (Grasselli et al., 2020; Salinas-Escudero et al., 2020; Tian et al., 2020).

Figura 3. Probabilidade de sobrevivência do infectado segundo a preexistência de comorbidades.

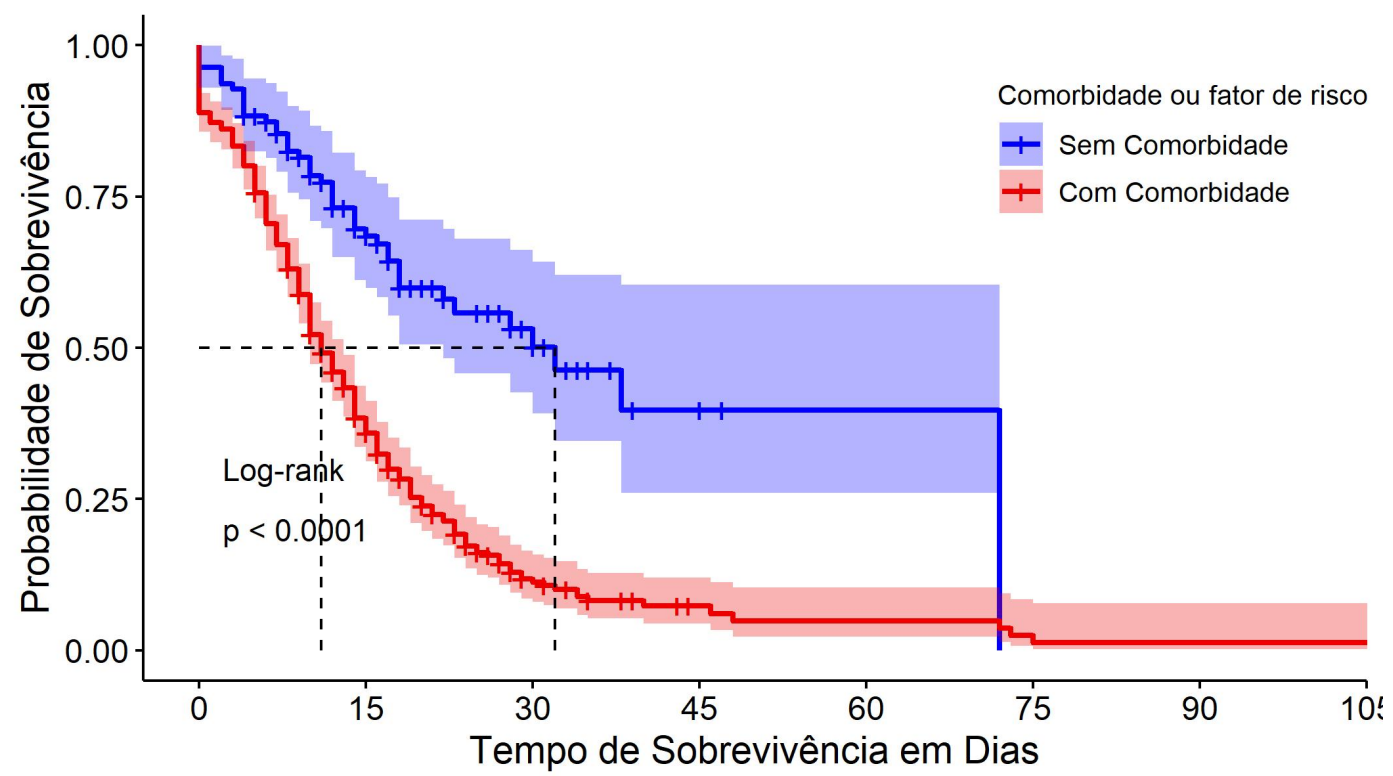

Fonte: Elaboração própria a partir dos microdados da SESAP-RN.

Nota: Dados obtidos em 13 de junho de 2020, sujeitos a atualizações.

A oferta de insumos hospitalares fornece a capacidade instalada para o atendimento e tratamento dos infectados pela COVID-19. Entretanto, como visto anteriormente, a distribuição de tais recursos físicos e humanos no território potiguar é desigual (Ministério da Saúde, 2020a; IPEA, 2020). Dessa forma, visando testar a hipótese de que o acesso a esses serviços de saúde tem correlação com a chance de sobrevivência do infectado pela COVID-19, a probabilidade de sobrevivência do infectado foi condicionada à Região de Saúde (RS) de residência da pessoa infectada. A Figura 4 ilustra a probabilidade de sobrevivência do infectado segundo a Região da Saúde de residência.

De fato, ao nível de 1\% de significância estatística, há diferença nas curvas de sobrevivência em relação à Região de Saúde. Na maior parte do período analisado, a probabilidade de sobrevivência é significativamente inferior para os indivíduos residentes na RS 6 (Pau dos Ferros). A RS 2 (Mossoró) é a que apresenta maior probabilidade de sobrevivência dos seus residentes. A mediana do tempo de sobrevivência para os residentes na RS 6 é de 7 dias, enquanto que a da RS 2 é de 16 dias, deixando evidente as disparidades entre as regiões de saúde. 
Figura 4. Probabilidade de sobrevivência do infectado segundo a Região de Saúde de residência

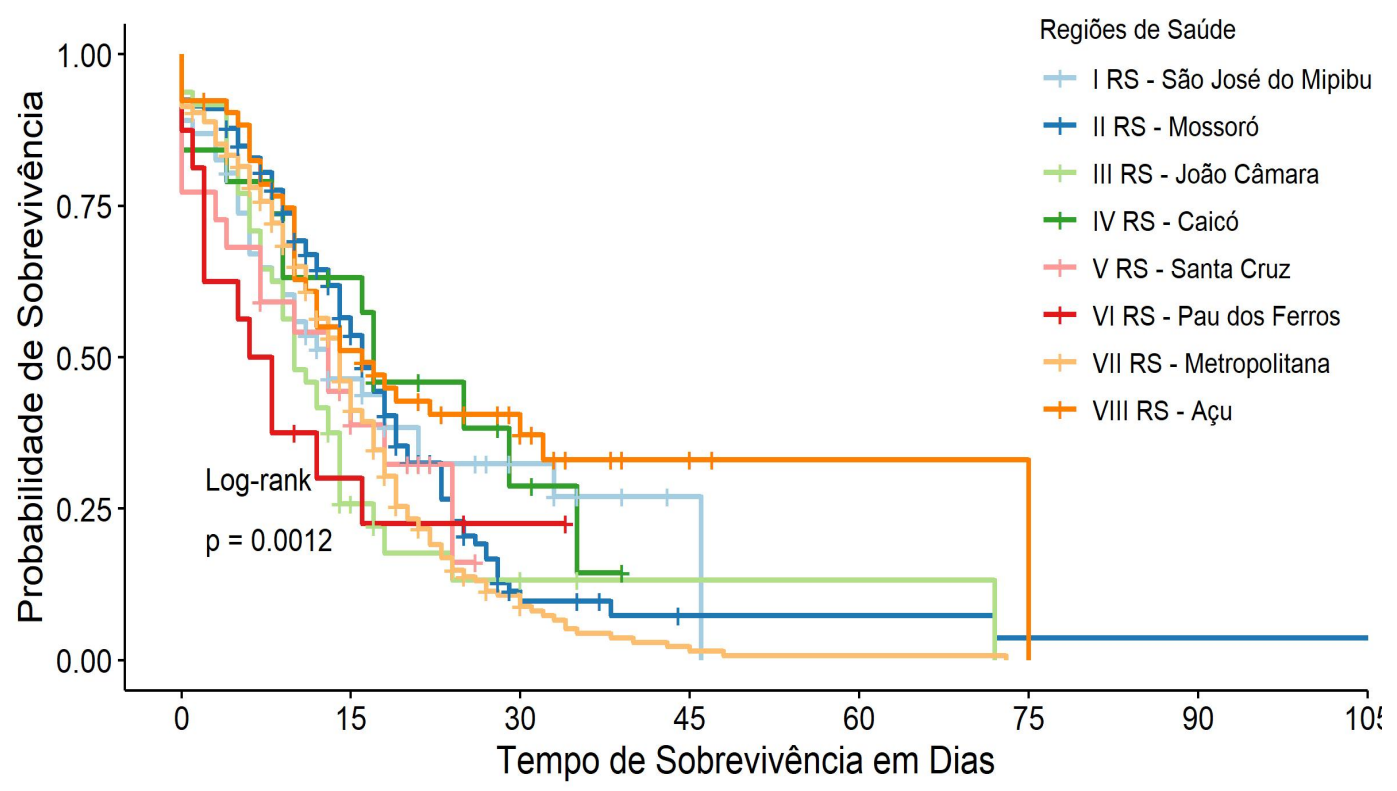

Fonte: Elaboração própria a partir dos microdados da SESAP-RN.

Nota: Dados obtidos em 13 de junho de 2020, sujeitos a atualizações.

Como visto anteriormente, a cor/raça/etnia do indivíduo infectado pela COVID19 pode ter uma correlação com o desfecho do caso (recuperação ou óbito) (Batista et al., 2020a). Dessa forma, estimou-se a função de sobrevivência condicionada à cor/raça/etnia declarada pelo indivíduo como visto na Figura 5 a seguir e verificouse que os pacientes autodeclarados da cor branca possuem maior probabilidade de sobreviver na comparação com os demais. Contudo, cabe destacar que a estatística de teste Log-rang foi considerada estatisticamente significante apenas ao nivel de $10 \%$.

Por fim, com o objetivo de verificar se o resultado encontrado nos estudos realizados por Grasselli et al. (2020), Nemati et al. (2020), Salinas-Escudero et al. (2020) e Tian et al. (2020) para a Itália, México e China, respectivamente, que sugerem que as chances de sobrevivência entre homens e mulheres são diferentes, se confirma no $\mathrm{RN}$, estimou-se a função de sobrevivência condicionada ao sexo, como apresentado na Figura 6.

Os resultados mostram que as curvas dos indivíduos do sexo feminino e do sexo masculino se encontram sobrepostas, dando indícios de que não há diferença na probabilidade de sobrevivência entre infectados dos dois gêneros no estado. A mediana do tempo de sobrevivência para o dois grupos é de aproximadamente 15 dias, sugerindo que também não há diferença no tempo de diferença para ambos os sexos. Estes resultados são confirmados pelo teste Log-rank que aponta para a não rejeição da hipótese nula de igualdade de probabilidade de sobrevivência, portanto, não há diferença estatística. Dentro desse contexto, o caso RN não corrobora com a evidência internacional, quando a condicionalidade é o sexo. 
Figura 5. Probabilidade de sobrevivência do infectado segundo a cor/raça/etnia

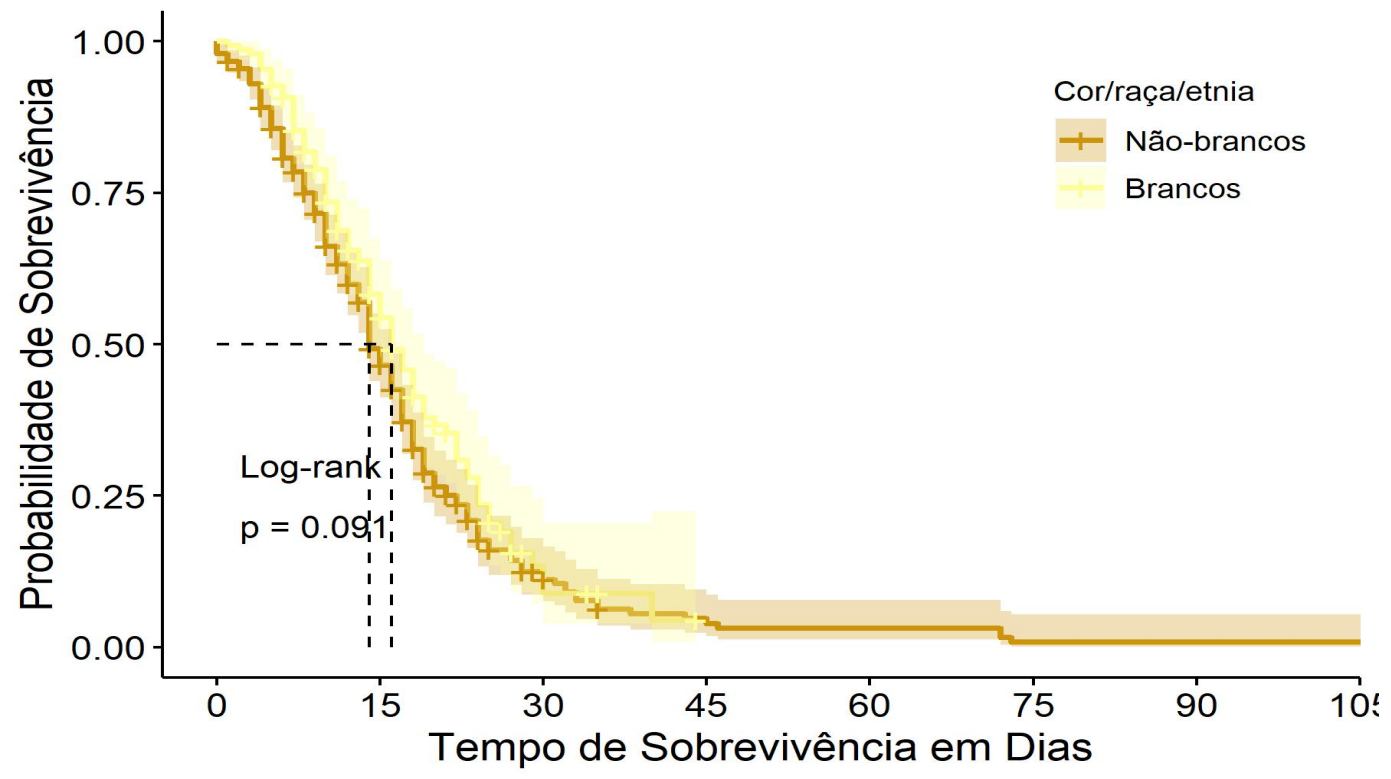

Fonte: Elaboração própria a partir dos microdados da SESAP-RN.

Nota: Dados obtidos em 13 de junho de 2020, sujeitos a atualizações.

Figura 6. Probabilidade de sobrevivência do infectado segundo o sexo.

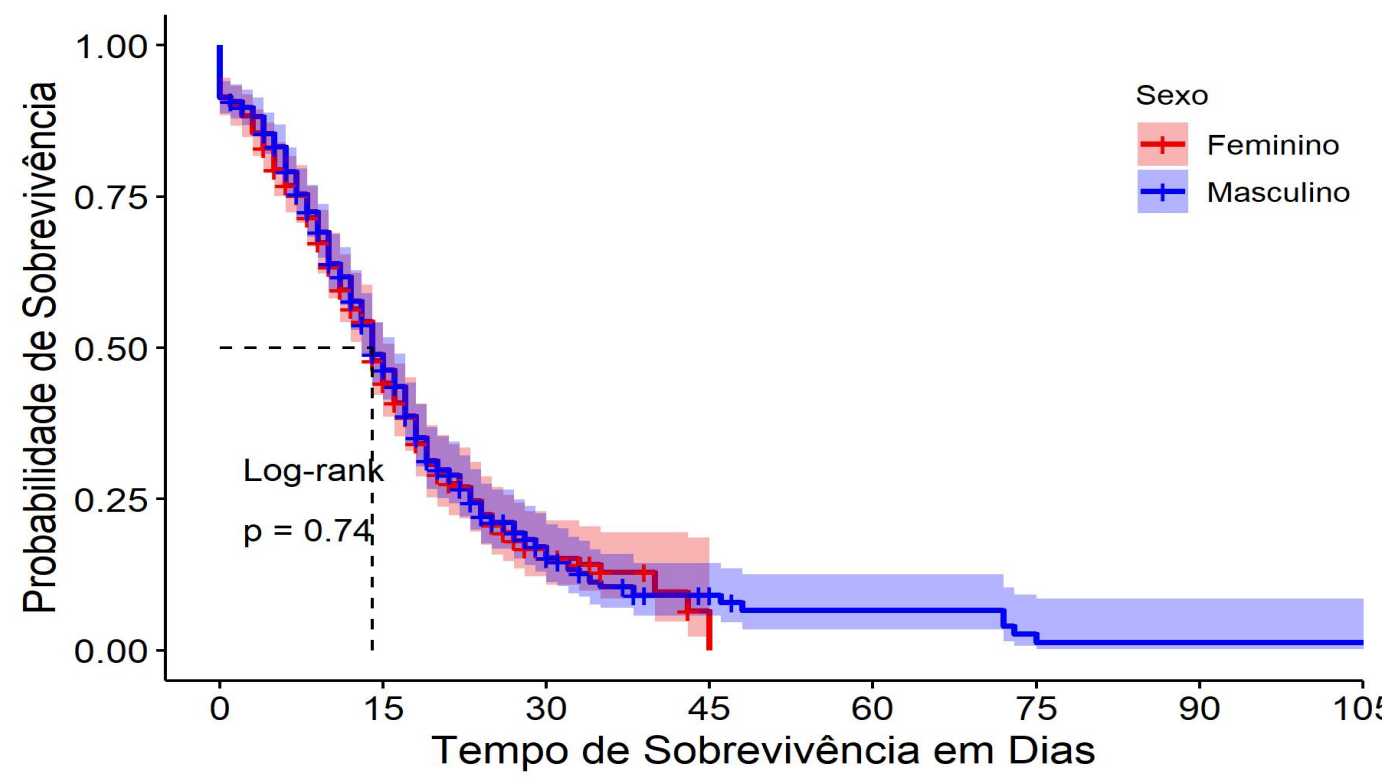

Fonte: Elaboração própria a partir dos microdados da SESAP-RN.

Nota: Dados obtidos em 13 de junho de 2020, sujeitos a atualizações.

Natal, capital do RN, concentra 38\% dos casos de COVID-19 registrados e também 46\% dos leitos de UTI do Estado. Além disso, verifica-se uma distribuição espacial desigual de equipamentos hospitalares, leitos e respiradores na própria capital do Estado (IPEA, 2020). Dessa forma, visando testar a hipótese de que a desigualdade de acesso a esses serviços de saúde dentro da capital tem correlação com a chance de sobrevivência do infectado pelo novo coronavírus, a amostra foi restringida à cidade 
de Natal e a probabilidade de sobrevivência do infectado foi condicionada à zona de localização do bairro de residência declarado no atendimento médico.

Pode-se constatar a partir da Figura 7 que, em todo o período analisado, os infectados residentes nos bairros das zonas norte e oeste possuem menor probabilidade de sobreviver na comparação com aqueles que residem nas zonas sul e leste. A mediana de tempo de sobrevivência é de apenas 14 dias para os moradores das zonas norte e oeste, enquanto é de 15 e 18 dias para os moradores das zonas leste e sul de Natal, respectivamente.

Figura 7. Probabilidade de sobrevivência do infectado segundo a zona de localização do bairro de residência.

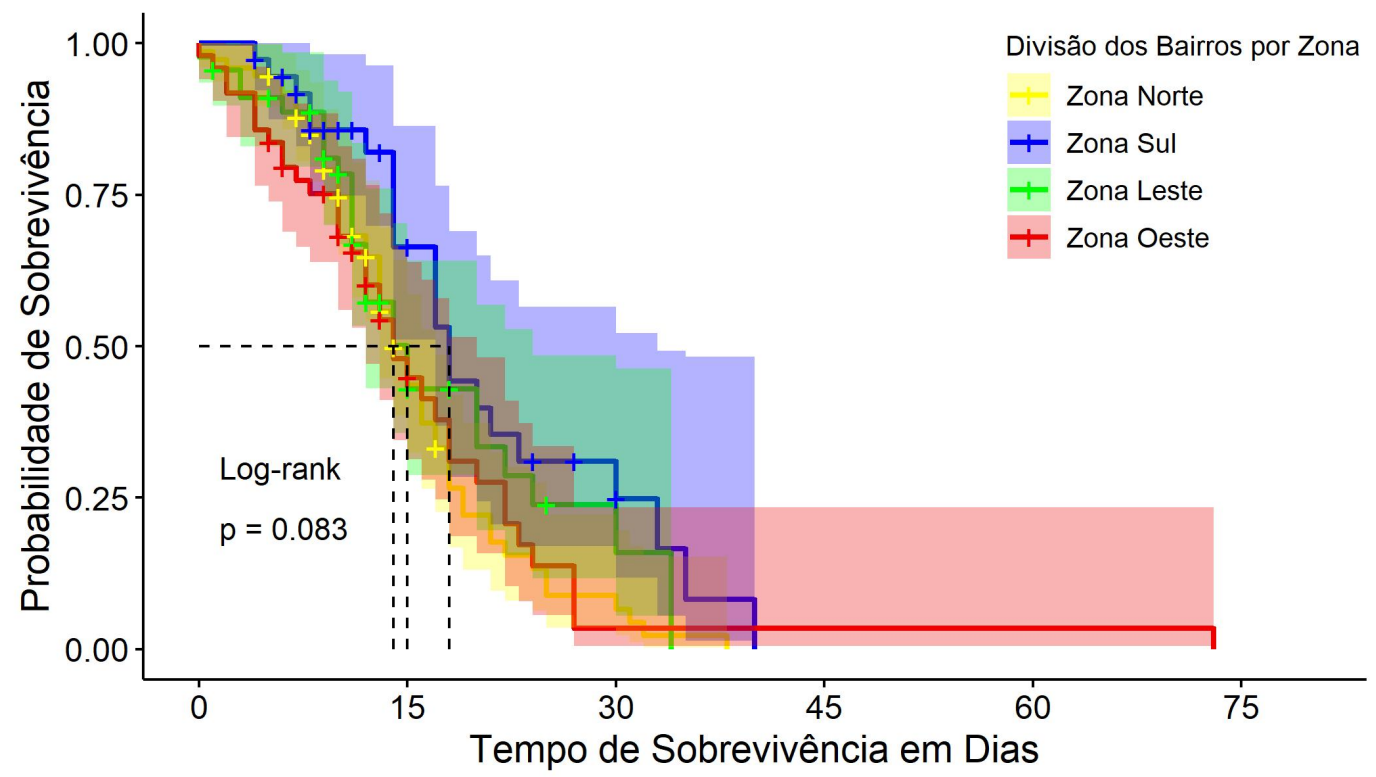

Fonte: Elaboração própria a partir dos microdados da SESAP-RN.

Nota: Dados obtidos em 13 de junho de 2020, sujeitos a atualizações.

Segundo o IPEA (2020), a zona oeste e a zona norte de Natal são as que mais concentram pessoas com dificuldade de acesso a estabelecimentos de saúde e hospitais na capital. De acordo com o estudo, é de 1,6 mil pessoas a população de baixa renda acima de 50 anos de idade de Natal que não consegue acessar nenhum estabelecimento de saúde em menos de 30 minutos de caminhada e 10,2 mil natalenses moram a uma distância maior do que $5 \mathrm{~km}$ do hospital mais próximo, com pelo menos um leito de UTI e um respirador. A zona norte, a mais populosa da cidade, conta com um número reduzido de respiradores, todos fornecidos pelo Sistema Único de Saúde (SUS). Um fator agravante é que nessas zonas existe uma grande concentração de pessoas de baixa renda, porém nelas residem um baixo percentual de pessoas idosas na comparação com as demais zonas, segundo dados do Censo 2010 (IBGE, 2010).

Em contraste, os bairros das zonas sul e leste de Natal possuem um percentual 
significativo de pessoas com rendimento acima de 5 salários mínimos - de acordo com os dados do Censo 2010 - e apresentam uma grande concentração de pessoas idosas (IBGE, 2010). Além disso, a distribuição espacial dos respiradores na cidade revela que eles estão bastante concentrados na zona leste, que também concentra boa parte dos equipamentos hospitalares.

A desigualdade de acesso aos serviços de saúde entre as zonas da cidade é confirmada pelo resultado do teste Log-rank que aponta para a existência de diferenças de chances de sobrevivência a depender do bairro de residência do infectado, ao nível de $10 \%$ de significância estatística, confirmando o resultado dos estudos realizados por Cobre et al. (2020), Demenech et al. (2020), Salinas-Escudero et al. (2020) e Santos et al. (2020) da existência de correlação positiva entre a desigualdade econômica e o risco de infecção e morte por COVID-19.

A seguir, serão apresentados os resultados do modelo de regressão Cox (1972), que analisa os fatores determinantes do evento óbito dos infectados pela COVID-19 no Rio Grande do Norte, quais sejam: sexo, cor, idade, tipo de comorbidade, tempo de diagnóstico e Região de Saúde. Os coeficientes estimados foram reportados na Tabela 3. As hipóteses nulas dos testes de Concordance, Likehood ratio e Wald foram rejeitadas, o que indica que as covariadas ajudam a explicar a ocorrência do evento morte pelo novo coronavírus.

O coeficiente associado à variável individual sexo (dummy que atribui valor igual a 1 quando o infectado for homem) não foi estatisticamente significativo, o que indica que o gênero parece não influenciar a probabilidade de morte. Esse resultado não corrobora com os resultados dos estudos de Cobre et al. (2020), Grasselli et al. (2020), Nemati et al. (2020), Salinas-Escudero et al. (2020) e Tian et al. (2020) de que homens tem maior probabilidade de morrer do que mulheres. O coeficiente da variável cor (dummy que atribui valor igual a 1 quando o infectado for branco) mostrou-se significativo, sugerindo que indivíduos da cor branca possuem menor probabilidade de óbito no estado, confirmando um padrão de letalidade da COVID-19 no RN similar ao do Brasil, como releva o estudo de Batista et al. (2020a). É importante ressaltar que a cor esconde uma correlação com a renda do indivíduo.

No que se refere à variável idade, observa-se que o sinal do coeficiente está de acordo com o esperado, visto que, foi observada uma relação positiva entre a idade e a probabilidade de morte confirmando os resultados dos estudos empíricos e esperado por especialistas em saúde (Batista et al., 2020a; China CDC Weekly, 2020; Cobre et al., 2020; Grasselli et al., 2020; Salinas-Escudero et al., 2020; Tian et al., 2020). O coeficiente de idade indica que 1 ano a mais de idade aumenta em 1,02 vezes o risco de morte do infectado. Este resultado se explica pelo fato dos idosos apresentarem um estado de saúde mais vulnerável do que os jovens já que a idade avançada geralmente está associada ao aparecimento de comorbidades, como hipertensão, diabetes, obesidade, entre outras, que podem os levar a morte, uma vez infectados. 
Tabela 3. Resultados das estimações do modelo Cox para a Probabilidade de Óbito do Infectado

\begin{tabular}{|c|c|c|c|c|c|c|}
\hline \multirow[t]{2}{*}{ Variáveis } & \multirow{2}{*}{$\begin{array}{l}\text { Modelo 1 } \\
\text { Coeficiente } \\
\text { (Erro- } \\
\text { padrão) }\end{array}$} & \multirow[b]{2}{*}{$\operatorname{Exp}($ Coef $)$} & \multirow{2}{*}{$\begin{array}{l}\text { Modelo } 2 \\
\text { Coeficiente } \\
\text { (Erro- } \\
\text { padrão) }\end{array}$} & \multirow{2}{*}{\multicolumn{2}{|c|}{$\begin{array}{cc} & \text { Modelo 3 } \\
\text { Exp(Coef) } & \text { Coeficiente } \\
& \text { (Erro- } \\
& \text { padrão) }\end{array}$}} & \multirow[b]{2}{*}{$\operatorname{Exp}($ Coef $)$} \\
\hline & & & & & & \\
\hline Homem & $\begin{array}{c}0,11 \\
(0,12)\end{array}$ & 1,12 & $\begin{array}{c}0,11 \\
(0,12)\end{array}$ & 1,12 & $\begin{array}{c}0,09 \\
(0,12)\end{array}$ & 1,10 \\
\hline Branco & $\begin{array}{l}-0,19 \\
(0,13)\end{array}$ & 0,83 & $\begin{array}{l}-0,22^{*} \\
(0,13)\end{array}$ & 0,80 & $\begin{array}{l}-0,22^{*} \\
(0,13)\end{array}$ & 0,80 \\
\hline Idade & $\begin{array}{l}0,02^{* * *} \\
(0,003)\end{array}$ & 1,02 & $\begin{array}{l}0,02^{* * *} \\
(0,003)\end{array}$ & 1,02 & $\begin{array}{l}0,02^{* * *} \\
(0,003)\end{array}$ & 1,02 \\
\hline HAS & $\begin{array}{c}0,50^{* * *} \\
(0,15)\end{array}$ & 1,65 & - & - & - & - \\
\hline Obesidade & - & - & $\begin{array}{l}0,43^{* *} \\
(0,21)\end{array}$ & 1,54 & - & - \\
\hline Renais crônicas & - & - & - & - & $\begin{array}{l}0,33^{*} \\
(0,20)\end{array}$ & 1,40 \\
\hline Tempo de diagnóstico & $\begin{array}{c}-0,11^{* * *} \\
(0,01)\end{array}$ & 0,90 & $\begin{array}{c}-0,10^{* * * *} \\
(0,01)\end{array}$ & 0,90 & $\begin{array}{c}-0,10^{* * *} \\
(0,01)\end{array}$ & 0,90 \\
\hline Região de Saúde 2 & $\begin{array}{c}-0,89^{* * *} \\
(0,24)\end{array}$ & 0,41 & $\begin{array}{c}-0,81^{* * *} \\
(0,24)\end{array}$ & 0,45 & $\begin{array}{c}-0,88^{* * *} \\
(0,24)\end{array}$ & 0,41 \\
\hline Região de Saúde 3 & $\begin{array}{l}-0,03 \\
(0,29)\end{array}$ & 0,97 & $\begin{array}{c}0,03 \\
(0,30)\end{array}$ & 1,03 & $\begin{array}{l}-0,06 \\
(0,29)\end{array}$ & 0,95 \\
\hline Região de Saúde 4 & $\begin{array}{l}-0,63 \\
(0,44)\end{array}$ & 0,53 & $\begin{array}{l}-0,58 \\
(0,45)\end{array}$ & 0,56 & $\begin{array}{l}-0,69 \\
(0,44)\end{array}$ & 0,50 \\
\hline Região de Saúde 5 & $\begin{array}{l}-0,77^{*} \\
(0,42)\end{array}$ & 0,46 & $\begin{array}{l}-0,77^{*} \\
(0,43)\end{array}$ & 0,46 & $\begin{array}{l}-0,99 * \\
(0,43)\end{array}$ & 0,37 \\
\hline Região de Saúde 6 & $\begin{array}{c}0,32 \\
(0,50)\end{array}$ & 1,38 & $\begin{array}{c}0,33 \\
(0,50)\end{array}$ & 1,39 & $\begin{array}{c}0,23 \\
(0,50)\end{array}$ & 1,26 \\
\hline Região de Saúde 7 & $\begin{array}{c}-0,87^{* * *} \\
(0,24)\end{array}$ & 0,42 & $\begin{array}{c}-0,82^{* * * *} \\
(0,25)\end{array}$ & 0,44 & $\begin{array}{c}-0,92^{* * *} \\
(0,24)\end{array}$ & 0,40 \\
\hline Região de Saúde 8 & $\begin{array}{l}-0,60^{*} \\
(0,31) \\
\end{array}$ & 0,55 & $\begin{array}{l}-0,53^{*} \\
(0,31) \\
\end{array}$ & 0,59 & $\begin{array}{c}-0,61^{* *} \\
(0,31)\end{array}$ & 0,54 \\
\hline $\mathrm{N}$ & 502 & & 502 & & 502 & \\
\hline № Eventos & 350 & & 350 & & 350 & \\
\hline Concordance & 0,72 & & 0,72 & & 0,72 & \\
\hline Likelihood ratio test & $185,4^{* * *}$ & & $179,3^{* * *}$ & & $178,1^{* * *}$ & \\
\hline Wald test & $131,1^{* * *}$ & & $124,4^{* * *}$ & & $123^{* * *}$ & \\
\hline Score (Logrank) Test & $123,1^{* * *}$ & & $116,8^{* * *}$ & & $116,6^{* * *}$ & \\
\hline
\end{tabular}

Fonte: Elaboração própria a partir dos microdados da SESAP.

Nota: Dados obtidos em 13 de junho de 2020, sujeitos a atualizações.

Nota 1: Nivel de significância: *** $0.01 * * 0.05 * 0.1$ " 1

Do mesmo modo, os sinais positivos e significativos dos coeficientes das variáveis de HAS, obesidade e doenças renais crônicas sugerem uma relação direta entre a preexistência dessas comorbidades e a probabilidade do infectado evoluir a óbito. $\mathrm{O}$ coeficiente da dummy de HAS indica que o diagnosticado anteriormente com hipertensão arterial sistêmica possui um risco de morte 1,65 vezes maior do que o indivíduo 
que não apresenta tal comorbidade. Similarmente, os coeficientes das dummies de obesidade e doenças renais crônicas indicam que indivíduos que apresentam tais comorbidades possuem um risco de morte 1,54 e 1,40 vezes maior, respectivamente, do que os infectados que não são acometidos com tais doenças. Tais resultados corroboram com as evidências encontradas nos estudos empíricos de que a presença de tais comorbidades no paciente coloca-o no grupo de risco, em vista do maior risco de morte se infectado pelo novo coronavírus (China CDC Weekly, 2020; Grasselli et al., 2020; Salinas-Escudero et al., 2020; Tian et al., 2020; Yang et al., 2020). Grasselli et al. (2020) acrescenta que uso de medicações contínuas como estatinas, diuréticos, entre outros, estão associados a um maior risco de mortalidade de infectados por COVID-19.

No que diz respeito ao tempo de diagnóstico, o coeficiente estimado sugere uma relação inversa entre o tempo de diagnóstico da COVID-19 e a probabilidade do infectado evoluir a óbito. Conforme Cobre et al. (2020) e Lima et al. (2021), como pode-se argumentar que quanto mais rápido o diagnóstico, mais rápido e eficaz o tratamento, impedindo que o quadro de saúde do acometido pela doença venha a se agravar e leve-o a morte, esse resultado pode parecer contraditório.

No entanto, o sinal na direção contrária do esperado para o coeficiente do tempo de diagnóstico pode ser resultado da escassez de testes capaz de diagnosticar a contaminação do paciente pela COVID-19. Isso porque, inicialmente, se testava somente os pacientes sintomáticos e, além disso, os pacientes do grupo de risco eram prioridades para a realização do teste. Outra possivel explicação pode ser a inadequação no tempo de realização dos testes para COVID-19, como aponta Lima et al. (2021) em seu estudo para as capitais brasileiras e Distrito Federal.

Os coeficientes negativos e estatisticamente significativos associados às Regiões de Saúde 2 e 7 sugerem que as características locais importam. É possível que esse resultado seja explicado pela distribuição desigual dos insumos hospitalares, que estão concentrados nas duas regiões citadas, especificamente nos municípios de Natal e Mossoró, sendo os dois municípios responsáveis por 66\% do total de leitos de UTI e 79\% dos respiradores do Estado (Ministério da Saúde, 2020a). Sendo assim, provavelmente os moradores das RS 2 e RS 7 possuam maior acesso a serviços de saúde mais complexos devido à proximidade a hospitais de referência que dispõem de UTI e respiradores para o tratamento dos casos graves e críticos da COVID-19 e, devido a isso, possuam maiores chances de sobrevivência IPEA (2020). Esses resultados estão de acordo com os achados empíricos de Cobre et al. (2020), Demenech et al. (2020) e Salinas-Escudero et al. (2020) de que residir em localidades com baixo índice de desenvolvimento social, a desigualdade econômica e a atenção à saúde no serviço público são fatores que elevam o risco de morte dos infectados.

Os resultados dos testes de diagnóstico da influência de outliers a partir do exame dos resíduos do modelo Cox constataram que nenhuma observação isoladamente influencia as demais de maneira significativa. 


\section{Considerações finais}

O objetivo central deste estudo consistiu em estimar a probabilidade e o tempo de sobrevivência dos infectados pela COVID-19 no estado do Rio Grande do Norte condicionados às características individuais, condição de saúde e Região de Saúde do indivíduo. Para esse fim, foi utilizada a técnica de análise de sobrevivência por meio da estimação da função de sobrevivência através do método Kaplan e Meier (1958) e do modelo Cox (1972) de risco, a exemplo do que foi realizado por Nemati et al. (2020) e Tian et al. (2020) utilizando dados de infectados na China.

Os resultados da análise de sobrevivência apontaram uma queda na probabilidade de sobrevivência do infectado com a COVID-19 com o passar do tempo, sugerindo que, com o passar dos dias, os sintomas dos infectados tendem a se agravar e levá-los a óbito, ao despeito da relação positiva encontrada entre a probabilidade de sobrevivência e o tempo de internação no estudo de Nemati et al. (2020) para pacientes com Síndrome Respiratória Aguda Grave (SRAG) na China.

Os resultados obtidos da estimação de funções de sobrevivência apontaram que há diferenças estatisticamente significativas na probabilidade e no tempo de sobrevivência em relação à faixa etária, preexistência ou não de comorbidades, Região de Saúde e zona do bairro de residência dos infectados. Indivíduos com idade maior ou igual a 60 anos, com comorbidades, residentes em regiões de saúde e bairros menos assistidos em termos de serviços de saúde apresentam menor probabilidade e tempo de sobrevivência.

Quanto aos resultados da estimação do modelo de risco, os coeficientes estatisticamente significantes das variáveis idade, de comorbidades como hipertensão, obesidade e doenças renais crônicas, do tempo de diagnóstico e das regiões de saúde de residência revelam que as características individuais, a condição de saúde e de acesso aos serviços de saúde podem ser determinantes para a sobrevivência do indivíduo infectado pela COVID-19 no Rio Grande do Norte, corroborando com as evidências nacionais (Cobre et al., 2020; Demenech et al., 2020; Santos et al., 2020) e internacionais (China CDC Weekly, 2020; Grasselli et al., 2020; Nemati et al., 2020; Salinas-Escudero et al., 2020; Tian et al., 2020; Yang et al., 2020).

Sendo assim, o estudo traz uma importante contribuição para efeito de formulação de políticas públicas ao concluir que, para além das características próprias do novo coronavírus, os aspectos individuais e regionais também podem ser determinantes para explicar a sobrevivência de um paciente contaminado pela COVID-19. Foi observado que as características populacionais, demográficas e socioeconômicas de uma localidade podem contribuir para aumentar a vulnerabilidade da população ao vírus e o risco de colapso do sistema de saúde. Dessa forma, é preciso levar em conta os indicadores socioeconômicos de cada localidade visando atender àquela determinada realidade social e reduzir o número de mortes. 
De posse da informação dos fatores determinantes para a sobrevivência e do tempo de recuperação/alta dos infectados pela COVID-19 é possivel um melhor planejamento das ações de combate à pandemia como, por exemplo, alocar de maneira mais eficiente os equipamentos hospitalares, leitos de UTI e respiradores que, por sua vez, ainda se encontram muito concentrados em certas regiões do Estado e localidades da própria capital, ao contrário da incidência da doença que passa por um processo de interiorização e espraiamento no território. Uma distribuição espacial mais igualitária dos recursos hospitalares entre as regiões de saúde do Estado e zonas da capital garantiria a igualdade de oportunidade de acesso aos serviços de saúde, aumentando as chances de sobrevivência dessa parcela da população desassistida.

Contudo, é importante salientar que o estudo possui algumas limitações. A primeira se refere à subnotificação de infectados e, principalmente, recuperados devido à baixa testagem da população e a falta de acompanhamento médico dos infectados em tratamento domiciliar. Do mesmo modo, a delimitação espacial da análise para o Estado do Rio Grande do Norte limita a interpretação dos resultados obtidos para esse Estado, que possui características populacionais, demográficas e socioeconômicas destoantes dos demais, e a comparação com outros estudos. Além disso, devido à atualização dos dados da SESAP-RN, a análise considerou apenas os casos confirmados de COVID-19 no Estado do Rio Grande do Norte até a data de 13 de Junho de 2020 , consequentemente acabou por censurar aqueles infectados que se recuperaram ou vieram a óbito após essa data.

\section{Referências}

Batista, A., Antunes, B., Faveret, G., Peres, I., Marchesi, J., Cunha, J. P., Dantas, L., Bastos, L., Carrilho, L., Aguilar, S., Baião, F., Maçaira, P., Hamacher, S., e Bozza, F. (2020a). Análise socioeconômica da taxa de letalidade da COVID-19 no Brasil. Nota Técnica 11, Núcleo de Operações e Inteligência em Saúde (NOIS).

Batista, A., Antunes, B., Peres, I., Marchesi, J., Cunha, J. P., Dantas, L., Bastos, L., Aguilar, S., Baião, F., Maçaira, P., Hamacher, S., e Bozza, F. (2020b). Evolução da epidemia de COVID-19 no Brasil até 04 de maio de 2020. Nota Técnica 10, Núcleo de Operações e Inteligência em Saúde (NOIS).

Castelnuovo, A. D., Bonaccio, M., Costanzo, S., Gialluisi, A., Antinori, A., Berselli, N., Blandi, L., Bruno, R., Cauda, R., Guaraldi, G., My, I., Menicanti, L., Parruti, G., Patti, G., Perlini, S., Santilli, F., Signorelli, C., Stefanini, G. G., Vergori, A., Abdeddaim, A., Ageno, W., Agodi, A., Agostoni, P., Aiello, L., Moghazi, S. A., Aucella, F., Barbieri, G., Bartoloni, A., Bologna, C., Bonfanti, P., Brancati, S., Cacciatore, F., Caiano, L., Cannata, F., Carrozzi, L., Cascio, A., Cingolani, A., Cipollone, F., Colomba, C., Crisetti, A., Crosta, F., Danzi, G. B., D’Ardes, D., de Gaetano Donati, K., Gennaro, F. D., Palma, G. D., Tano, G. D., Fantoni, M., Filippini, T., Fioretto, P., Fusco, F. M., Gentile, I., Grisafi, L., Guarnieri, G., Landi, F., Larizza, G., Leone, 
A., Maccagni, G., Maccarella, S., Mapelli, M., Maragna, R., Marcucci, R., Maresca, G., Marotta, C., Marra, L., Mastroianni, F., Mengozzi, A., Menichetti, F., Milic, J., Murri, R., Montineri, A., Mussinelli, R., Mussini, C., Musso, M., Odone, A., Olivieri, M., Pasi, E., Petri, F., Pinchera, B., Pivato, C. A., Pizzi, R., Poletti, V., Raffaelli, F., Ravaglia, C., Righetti, G., Rognoni, A., Rossato, M., Rossi, M., Sabena, A., Salinaro, F., Sangiovanni, V., Sanrocco, C., Scarafino, A., Scorzolini, L., Sgariglia, R., Simeone, P. G., Spinoni, E., Torti, C., Trecarichi, E. M., Vezzani, F., Veronesi, G., Vettor, R., Vianello, A., Vinceti, M., Caterina, R. D., e Iacoviello, L. (2020). Common cardiovascular risk factors and in-hospital mortality in 3,894 patients with COVID 19: survival analysis and machine learning-based findings from the multicentre Italian CORIST Study. Nutrition, Metabolism and Cardiovascular Diseases, 30(11):1899-1913.

Cavalcanti, G. S. (2020). Ensaios em economia da educação. Mestrado em economia, Programa de Pós-Graduação em Economia, Universidade Federal do Rio Grande do Norte, Natal.

China CDC Weekly (2020). The epidemiological characteristics of an outbreak of 2019 novel coronavirus diseases (COVID-19) - China, 2020. China CDC Weekly, 2(8):113-122.

Cobre, A. d. F., Böger, B., Fachi, M. M., Vilhena, R. d. O., Domingos, E. L., Tonin, F. S., e Pontarolo, R. (2020). Risk factors associated with delay in diagnosis and mortality in patients with COVID-19 in the city of Rio de Janeiro, Brazil. Ciencia \& Saúde Coletiva, 25:4131-4140.

Cox, D. R. (1972). The analysis of multivariate binary data. 21:113.

Delatorre, E., Mir, D., Gräf, T., e Bello, G. (2020). Tracking the onset date of the community spread of SARS-CoV-2 in western countries. Memorias do Instituto Oswaldo Cruz, 115:e200183.

Demenech, L. M., Dumith, S. d. C., Vieira, M. E. C. D., e Neiva-Silva, L. (2020). Income inequality and risk of infection and death by COVID-19 in Brazil. Revista brasileira de Epidemiologia, 23:e200095.

DeWitte, S. N. (2014). Mortality risk and survival in the aftermath of the medieval Black Death. PloS One, 9:e96513.

Fio Cruz (2020). Interiorização do Covid-19 e as redes de atendimento em saúde. Nota Técnica 02 de maio de 2020, MonitoraCovid-19 - FIOCRUZ.

FMI (2020). Novas previsões sugerem uma recessão mais profunda e uma recuperação mais lenta. Texto para discussão, World Economic Forum.

Grasselli, G., Greco, M., Zanella, A., Albano, G., Antonelli, M., Bellani, G., Bonanomi, E., Cabrini, L., Carlesso, E., Castelli, G., Cattaneo, S., Cereda, D., Colombo, S., Coluccello, A., Crescini, G., Forastieri Molinari, A., Foti, G., Fumagalli, R., Iotti, 
G. A., Langer, T., Latronico, N., Lorini, F. L., Mojoli, F., Natalini, G., Pessina, C. M., Ranieri, V. M., Rech, R., Scudeller, L., Rosano, A., Storti, E., Thompson, B. T., Tirani, M., Villani, P. G., Pesenti, A., Cecconi, M., e Network, C. O. V. I. D. L. I. C. U. (2020). Risk Factors Associated With Mortality Among Patients With COVID-19 in Intensive Care Units in Lombardy, Italy. JAMA Internal Medicine, 180:1345-1355.

Greene, W. H. (2008). Econometric analysis. Pearson Prentice-Hall, Upper Saddle River, 6 ed. edition.

IBGE (2010). Instituto brasileiro de geografia e estatística. censo demográfico 2010. https://www.ibge.gov.br/.

IBGE (2021). Instituto brasileiro de geografia e estatística. pesquisa nacional de saúde 2019: Percepção do estado de saúde, estilos de vida, doenças crônicas e saúde bucal. https://www.ibge.gov.br/.

IPEA (2020). Mobilidade urbana e o acesso ao Sistema Único de Saúde para casos suspeitos e graves de Covid-19 nas vinte maiores cidades do Brasil. Nota Técnica 14, Instituto de Pesquisa Econômica Aplicada, Brasília. Diretoria de Estudos e Políticas Regionais, Urbanas e Ambientais.

Jin, J.-M., Bai, P., He, W., Wu, F., Liu, X.-F., Han, D.-M., Liu, S., e Yang, J.-K. (2020). Gender differences in patients with COVID-19: Focus on severity and mortality. Frontiers in Public Health, 8.

Johnson, N. P.; Mueller, J. (2002). Updating the accounts: global mortality of the 1918-1920 "Spanish"influenza pandemic. Bulletin of the History of Medicine, 76(1):105-115.

Jones, K. E., Patel, N. G., Levy, M. A., Storeygard, A., Balk, D., Gittleman, J. L., e Daszak, P. (2008). Global trends in emerging infectious diseases. Nature, 451(7181):990-993.

Kaplan, E. L. e Meier, P. (1958). Nonparametric estimation from incomplete observations. Journal of the American Statistical Association, 53:457-481.

Kleinbaum, D. G. e Klein, M. (2012). Survival analysis: A self-learning text.

LAIS-UFRN (2020). Laboratório de inovação tecnológica em saúde. https://covid.lais.ufrn.br/. Acesso em: 13 jun. 2020.

Li, X., Xu, S., Yu, M., Wang, K., Tao, Y., Zhou, Y., Shi, J., Zhou, M., Wu, B., Yang, Z., Zhang, C., Yue, J., Zhang, Z., Renz, H., Liu, X., Xie, J., Xie, M., e Zhao, J. (2020). Risk factors for severity and mortality in adult COVID-19 inpatients in Wuhan. Journal of Allergy and Clinical Immunology, 146(1):110-118.

Lichtenthäler Filho, R. e Lichtenthäler, D. G. (2020). A dynamic model for Covid-19 in Brazil. Cold Spring Harbor Laboratory. 
Lima, F. E. T., de Albuquerque, N. L. S., de Souza Gurgel Florencio, S., Fontenele, M. G. M., Queiroz, A. P. O., Lima, G. A., de Figueiredo, L. M., Amorim, S. M. C., e Barbosa, L. P. (2021). Intervalo de tempo decorrido entre o início dos sintomas e a realização do exame para COVID-19 nas capitais brasileiras, agosto de 2020 . Epidemiologia e Serviços de Saúde, 30(1).

Madhav, N., Oppenheim, B., Gallivan, M., Mulembakani, P., Rubin, E., e Wolfe, N. (2017). Pandemics: Risks, impacts, and mitigation. In: Disease control priorities: Improving Health and Reducing Poverty, volume 9. World Bank, 3 ed. edition.

Mckibbin, W. J.; Sidorenko, A. A. (2006). Global Macroeconomic Consequences of Pandemic Influenza. Lowy Institute for International Policy, Sydney, Australia.

Ministério da Saúde (2020a). Cadastro nacional de estabelecimentos de saúde - datasus. http://cnes.datasus.gov.br/.

Ministério da Saúde (2020b). Painel coronavírus. https://covid.saude.gov.br/. Acesso em: 13 jun. 2020.

Morse, J. M. (1995). The significance of saturation. SAGE Social Science.

Nemati, M., Ansary, J., e Nemati, N. (2020). COVID-19 machine learning based survival analysis and discharge time likelihood prediction using clinical data. SSRN Electronic Journal.

Noronha, K. V. M. d. S., Guedes, G. R., Turra, C. M., Andrade, M. V., Botega, L., Nogueira, D., Calazans, J. A., Carvalho, L., Servo, L., e Ferreira, M. F. (2020). Pandemia por COVID-19 no Brasil: análise da demanda e da oferta de leitos hospitalares e equipamentos de ventilação assistida segundo diferentes cenários. Cadernos de Saúde Pública, 36(6).

Parpia, A. S., Ndeffo-Mbah, M. L., Wenzel, N. S., e Galvani, A. P. (2016). Effects of response to 2014-2015 ebola outbreak on deaths from malaria, HIV/AIDS, and tuberculosis, West Africa. Emerging Infectious Diseases, 22(3):433-441.

R Core Team (2019). R: A language and environment for statistical computing. $R$ Foundation for Statistical Computing. https://www.R-project.org/.

Rio Grande do Norte (2020). Boletim Epidemiológico № 87. techreport 87, Secretaria de Estado da Saúde Pública, Natal.

Salinas-Escudero, G., Carrillo-Vega, M. F., Granados-García, V., Martínez-Valverde, S., Toledano-Toledano, F., e Garduño-Espinosa, J. (2020). A survival analysis of COVID-19 in the Mexican population. BMC Public Health, 20(1).

Santos, J. P. C., Siqueira, A. S. P., Praça, H. L. F., e Albuquerque, H. G. (2020). Vulnerabilidade a formas graves de COVID-19: uma análise intramunicipal na cidade do Rio de Janeiro, Brasil. Cadernos de Saúde Pública, 36(5). 
Senhoras, E. M. (2020). CoronavÎrus e o papel das pandemias na história humana.

Tian, R., Wu, W., Wang, C., Pang, H., Zhang, Z., Xu, H., Luo, Q., Gao, P., Shi, J., Li, W., Gian, H., Guo, F., Li, T., Liu, Z., Wang, J., Zhou, X., Qin, Y., Yan, X., e Zhang, S. (2020). Clinical characteristics and survival analysis in critical and non-critical patients with COVID-19 in Wuhan, China: a single-center retrospective case control study. Scientific Reports, 10(1).

Wooldridge, J. (2002). Econometric analysis of cross section and panel data. MIT Press Books, Cambridge.

Wu, F., Zhao, S., Yu, B., Chen, Y.-M., Wang, W., Song, Z.-G., Hu, Y., Tao, Z.-W., Tian, J.-H., Pei, Y.-Y., Yuan, M.-L., Zhang, Y.-L., Dai, F.-H., Liu, Y., Wang, Q.-M., Zheng, J.-J., Xu, L., Holmes, E. C., e Zhang, Y.-Z. (2020). A new coronavirus associated with human respiratory disease in China. Nature, 579(7798):265-269.

Yang, J., Zheng, Y., Gou, X., Pu, K., Chen, Z., Guo, Q., Ji, R., Wang, H., Wang, Y., e Zhou, Y. (2020). Prevalence of comorbidities and its effects in patients infected with SARS-CoV-2: a systematic review and meta-analysis. International Journal of Infectious Diseases, 94:91-95.

Zhou, P., Yang, X.-L., Wang, X.-G., Hu, B., Zhang, L., Zhang, W., Si, H.-R., Zhu, Y., Li, B., Huang, C.-L., Chen, H.-D., Chen, J., Luo, Y., Guo, H., Jiang, R.-D., Liu, M.-Q., Chen, Y., Shen, X.-R., Wang, X., Zheng, X.-S., Zhao, K., Chen, Q.-J., Deng, F., Liu, L.-L., Yan, B., Zhan, F.-X., Wang, Y.-Y., Xiao, G.-F., e Shi, Z.-L. (2020). A pneumonia outbreak associated with a new coronavirus of probable bat origin. Nature, 579(7798):270-273.

(巛) Ev 